\title{
Speeding Up Object Detection Fast Resizing in the Integral Image Domain
}

\author{
Michael Gschwandtner, Andreas Uhl and Andreas Unterweger \\ Department of Computer Sciences, University of Salzburg, Jakob-Haringer-Straße 2, Salzburg, Austria \\ aunterweg@cosy.sbg.ac.at
}

Keywords:
Integral Image, Resizing, Object Detection, Performance.

Abstract:

In this paper, we present an approach to resize integral images directly in the integral image domain. For the special case of resizing by a power of two, we propose a highly parallelizable variant of our approach, which is identical to bilinear resizing in the image domain in terms of results, but requires fewer operations per pixel. Furthermore, we modify a parallelized state-ofthe-art object detection algorithm which makes use of integral images on multiple scales so that it uses our approach and compare it to the unmodified implementation. We demonstrate that our modification allows for an average speedup of $6.38 \%$ on a dual-core processor with hyperthreading and $12.6 \%$ on a 64 -core multi-processor system, respectively, without impacting the overall detection performance. Moreover, we show that these results can be extended to a whole class of object detection algorithms.

\section{INTRODUCTION}

Integral images, initially developed under the name "summed-area tables" (Crow, 1984), have regained a lot of attention since Viola and Jones proposed an object detection framework (Viola and Jones, 2001) which makes heavy use of them. Popular implementations of this framework, such as the OpenCV library (Willow Garage, 2012), perform object detection on multiple scales, i.e., they resize the original image multiple times and run the detection algorithm on each resized image, also referred to as scale. Although the object detection is relatively fast due to the use of integral images, the need to recompute the integral image for each scale impacts the performance significantly. Therefore, in this paper, we propose a new algorithm which allows resizing the integral images themselves, i.e., in the integral image domain instead of the image domain, omitting the need to recompute the integral image for each scale.

Despite efforts to speed up the computation of integral images in general (Hensley et al., 2005) as well as on different architectures like GPUs (Bilgic et al., 2010), literature on integral images is sparse. While Crow (Crow, 1984) initially described how to perform simple operations, like, e.g., blurring, in the integral image domain, Heckbert (Heckbert, 1986) generalized the underlying theory, thereby extending its scope to arbitrary filters with polynomial kernels. Hussein (Hussein et al., 2008) improved and extended Heckbert's work by enabling non-uniform filtering. Although both frameworks, Heckbert's and Hussein's, allow to perform resizing operations, they take input from the integral image domain and produce output in the image domain. In contrast, our approach performs all operations directly in the integral image domain, hereby omitting the need to recompute the integral image after resizing. Although algorithms performing operations directly in the integral image domain have been proposed (e.g., (Yu et al., 2010) for histogram thresholding), none of them changes the size of the integral image itself, as opposed to the algorithm we propose. As performing operations on multiple scales in the integral image domain is part of several state-of-the-art algorithms, such as SURF (Bay et al., 2008), local binary patterns (LBP) (Ahonen et al., 2004) and Viola's and Jones' framework for object detection as ex- 
plained above, our main contribution of resizing in the integral image domain inherently allows speeding up algorithms relying on the computation of integral images on multiple scales. Note that, although some algorithms allow scaling up the features instead of scaling down the integral images, a significant number of implementations (Willow Garage, 2012) recompute the integral images and thus profit from our contribution.

This paper is structured as follows: In section 2 , we propose an algorithm for resizing in the integral image domain without distortions by imposing certain restrictions on the resizing factor. In section 3, we extend this algorithm to support arbitrary resizing factors, albeit at the cost of negligible distortions. After evaluating our algorithm in section 4 in terms of performance, quality and parallelizability, we conclude our paper in section 5.

\section{EXACT RESIZING}

In the following sections we describe how a given integral image can be resized. We distinguish between exact and approximate resizing, where exact means that each pixel of the resized integral image is identical to the corresponding pixel of an integral image which is calculated from a bilinearly resized version of the original image, the resizing process of which has been performed in the image domain.

\subsection{Integral Images}

An integral image $I I$ of a given image $I$ represents the sum of all its pixels from the top-left corner to every pixel, excluding the column and row of the pixel (note that some definitions include the pixel's column and row, requiring corresponding changes in the subsequent formulas). Hence, it is calculated as (Willow Garage, 2012)

$$
I I(x, y)=\sum_{x^{\prime}=0}^{x-1} \sum_{y^{\prime}=0}^{y-1} I\left(x^{\prime}, y^{\prime}\right)
$$

This allows calculating the sum $S$ of all pixels within a rectangular area $R$ in constant time (Crow, 1984) as

$S_{R}=I I\left(x_{r}, y_{b}\right)-I I\left(x_{l}, y_{b}\right)-I I\left(x_{r}, y_{t}\right)+I I\left(x_{l}, y_{t}\right)$

where $x_{l}, x_{r}, y_{t}$ and $y_{b}$ are $R$ 's left, right, top and bottom coordinates, respectively, as depicted in figure 1. Note that, in order to reconstruct single

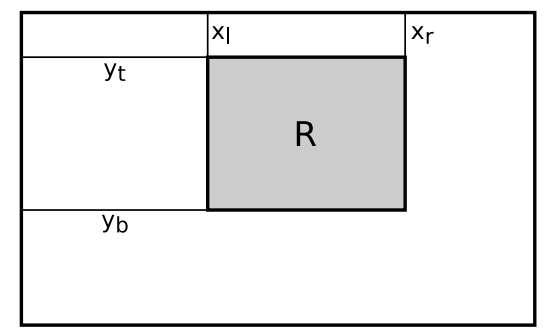

Figure 1: Use of an integral image for summing all pixels within a rectangular area $R$ constrained by its coordinates $x_{l}, x_{r}, y_{t}$ and $y_{b}$. Adopted from (Crow, 1984)

pixels from the integral image (see next section for details), the integral image's dimensions are $(w+1) \cdot(h+1)$ if the original image's dimensions are $w \cdot h$.

\section{$2.2 \quad$ Naïve Resizing}

Resizing algorithms usally perform operations in the image domain, i.e., on the image's pixels. As becomes clear from equation (2), it is possible to extract every single pixel as a rectangle of width and height one of the original image $I$ from its integral image $I I$ :

$$
\begin{aligned}
I(x, y)= & I I(x, y)+I I(x+1, y+1)- \\
& I I(x, y+1)-I I(x+1, y)
\end{aligned}
$$

Therefore, it is theoretically possible to implement any image-domain-based resizing filter in the integral image domain by filtering using onthe-fly extraction of the original image's pixels and subsequent calculation of the resized image's integral image.

However, this is computationally more expensive as accessing each pixel requires four operations in the integral image domain as opposed to one in the image domain. Furthermore, it is necessary to access locations in the integral image which are one row apart in order to derive a single pixel of the original image. This may cause a higher number of the CPU's cache lines to be occupied, if the integral image is stored sequentially in memory.

\subsection{Resizing By A Power Of Two}

In the following section we propose a resizing algorithm for integral images which eliminates the need to extract the original image's pixels from the integral image in a computationally expensive way. However, for the algorithm to work exactly, the resizing factor needs to be a power of two. 


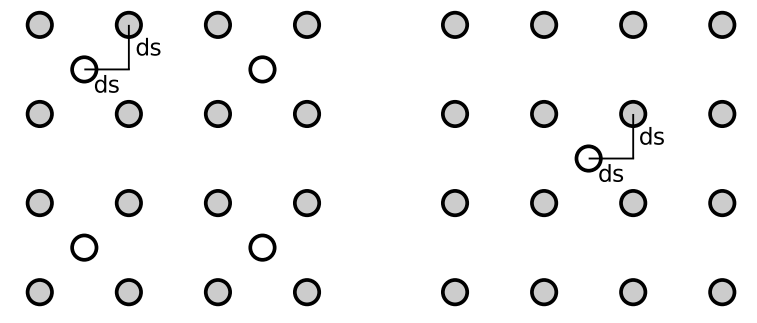

Figure 2: Resizing by a power of two using a special case of bilinear interpolation where the interpolated samples (white) have the same distance $d s$ to all surrounding original samples (gray).

Note that we discuss ways to circumvent this restriction in section 3 .

Consider the following, simplified resizing scenario: A given image $I$ with width $w$ and height $h$, where both, $w$ and $h$, are even, is to be resized by a factor of two in each dimension, yielding the image $I_{h}$ with width $\frac{w}{2}$ and height $\frac{h}{2}$. Using bilinear interpolation as depicted in figure 2 (left), the samples of $I$ (gray) are used to determine the samples of $I_{h}$ (white) as:

$$
\begin{aligned}
I_{h}(x, y)=\frac{1}{4} \cdot( & (2 x, 2 y)+I(2 x+1,2 y)+ \\
& I(2 x, 2 y+1)+I(2 x+1,2 y+1))
\end{aligned}
$$

The integral image $I I_{h}$ at position $(0 \leq x \leq$ $\frac{w}{2}, 0 \leq y \leq \frac{h}{2}$ ) of $I_{h}$ can then be calculated by

$$
I I_{h}(x, y)=\sum_{x^{\prime}=0}^{x-1} \sum_{y^{\prime}=0}^{y-1} I_{h}\left(x^{\prime}, y^{\prime}\right)
$$

which can be expanded to

$$
\begin{aligned}
\frac{1}{4} \cdot \sum_{x^{\prime}=0}^{x-1} \sum_{y^{\prime}=0}^{y-1} I\left(2 x^{\prime}, 2 y^{\prime}\right)+I\left(2 x^{\prime}+1,2 y^{\prime}\right)+ \\
I\left(2 x^{\prime}, 2 y^{\prime}+1\right)+I\left(2 x^{\prime}+1,2 y^{\prime}+1\right)
\end{aligned}
$$

This can be rewritten as

$$
I I_{h}(x, y)=\frac{1}{4} \cdot \sum_{x^{\prime}=0}^{2 x-1} \sum_{y^{\prime}=0}^{2 y-1} I\left(x^{\prime}, y^{\prime}\right)
$$

where the summand can subsequently be expressed as a sample of the integral image $I I$ of the original image $I$ :

$$
I I_{h}(x, y)=\frac{1}{4} \cdot I I(2 x, 2 y)
$$

Note that this equation only depends on the original image's integral image $I I$ and has no dependency to the original image $I$. Furthermore, it trivially allows repeated application (e.g., twice for a resizing factor of four as illustrated in figure 2 (right)), thereby enabling resizing by arbitrary powers of two. As can be easily shown, a given integral image $I I$ can be resized by a factor of $2^{n}$ in each dimension to an integral image $I I_{n}$ as

$$
I I_{n}(x, y)=\frac{1}{2^{2 n}} \cdot I I\left(2^{n} x, 2^{n} y\right)
$$

Based on this observation, we formulate our approach to resize integral images as follows: An integral image can be resized by a power of two with bilinear interpolation using only one single integral image sample per calculated sample using equation (9). Note that the latter assumes both, the corresponding image's width and height, to be integer multiples of $2^{n}$. For all other cases, resizing cannot be performed exactly at the integral image's borders. However, the handling of these borders in approximate form is described in section 3.2

\section{APPROXIMATE RESIZING}

In order to overcome the limitations of the resizing approach proposed in the previous section in terms of image dimensions and resizing factors, we present an extension which can deal with arbitrary resizing factors and image borders. Nonetheless, this extended approach is largely based on the limited approach presented in the previous section.

\subsection{Resizing Arbitrarily}

In this section we explain how to extend equation (8) in order to support arbitrary resizing factors. We do so by splitting the formula into two parts the factor in front of the sum and the sum itself. By modifying each of them separately, we derive an equation which can be used to resize arbitrarily in the integral image domain.

When resizing by a factor two in each dimension, we observe that the factor of $\frac{1}{4}$ in front of the sum in equation (8) corresponds to the inverse of the combined (i.e., multiplied) resizing factor. Simply put, each pixel of the resized image covers an area of 4 pixels in the original image, as depicted in figure 3 (left). This is equivalently true for the corresponding integral image pixels.

Extending this observation to arbitrary resizing factors, henceforth denoted as $2 a$, it is obvious that each pixel of the resized image now covers an 

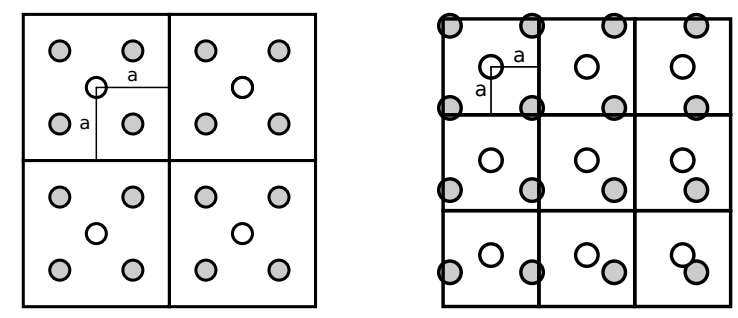

Figure 3: Resized (integral) image samples (white) covering a constant area of $4 a^{2}$ (black rectangle) in the original (integral) image (gray samples) when resizing by a factor of $2 a=2$ (left) and $2 a=1.16$ (right), respectively. For both, the resizing filter offset $b=0.5$ samples (see equation 10 ).

area of $2 a \cdot 2 a=4 a^{2}$ square pixels in the original image. Figure 3 (right) depicts this for a resizing factor of $2 a=1.16$, where the covered area is $4 a^{2}=1.3456$ square pixels. Note that each pixel of the resized image is located at the center of the area it covers in the original image, i.e., its distance to each side of the rectangle enclosing this area is $a$.

Although changing the factor in equation (8) to $\frac{1}{4 a^{2}}$ does not introduce an error, the required corresponding change of each summand does. Replacing $I I(2 x+1,2 y+1)$ by $I I(2 a x+b, 2 a y+b)$ (where $-a<b<a$ denotes a offset corresponding to the desired resizing filter phase, which is typically constant for all samples) is not possible in general, as $a$ is not necessarily an integer.

Therefore, we suggest performing bilinear interpolation in the integral image domain in order to get an approximation of the virtual pixel at position $I I(2 a x+b, 2 a y+b)$ based on the values of the surrounding integral image pixels. Note that this approximation introduces a small error compared to the bilinear interpolation in the image domain. This error is estimated empirically in section 4.2 . Summarizing the above modifications to equation (8), an integral image $I I$ can be resized by a factor of $2 a$ in both dimensions in the integral image domain in the same (mathematical) way as in the image domain, i.e., by bilinear interpolation. Doing so yields a resized integral image $I I_{r}$ which can be calculated by

$$
\begin{array}{r}
I I_{r}(x, y) \approx \frac{1}{4 a^{2}} \cdot \operatorname{bilinear}(I I,(2 a x+b, 2 a y+b)) \\
=\frac{1}{4 a^{2}} \cdot\left(\left[\begin{array}{ll}
1-d x & d x
\end{array}\right]\left[\begin{array}{ll}
i_{t l} & i_{b l} \\
i_{t r} & i_{b r}
\end{array}\right]\left[\begin{array}{c}
1-d y \\
d y
\end{array}\right]\right)
\end{array}
$$

for all values of $x$ and $y$ except the borders (see section 3.2 for details), where

$$
\begin{aligned}
i_{t l} & =I I\left(x^{\prime}, y^{\prime}\right), i_{t r}=I I\left(x^{\prime}+1, y^{\prime}\right) \\
i_{b l} & =I I\left(x^{\prime}, y^{\prime}+1\right), i_{b r}=I I\left(x^{\prime}+1, y^{\prime}+1\right) \\
x^{\prime} & =\lfloor 2 a x+b\rfloor, y^{\prime}=\lfloor 2 a y+b\rfloor \\
d x & =2 a x+b-x^{\prime}, d y=2 a y+b-y^{\prime}
\end{aligned}
$$

The value of $b$ has to be chosen according to the desired filter phase as explained above. Note that equation (10) uses the same formula for bilinear interpolation as any comparable algorithm in the image domain would. The only difference is that the latter operates on the image's pixels, while the former operates on the integral image's.

\subsection{Handling Of Borders}

For positive $b$, the rightmost column and the bottommost row can, in most cases, not be calculated by equation (10) as non-existing samples of the original integral image, i.e., samples whose coordinates are larger than the image's width and/or height, respectively, would have to be accessed. For negative $b$, the same applies to the leftmost column and the topmost row.

The latter case $(b<0)$ is trivial to handle: All samples can be set to zero as the first row and column of an integral image is by definition (see equation 1) zero. The former case $(b>0)$ can be handled in a way similar to the approach described in section 3.1. While the area covered by the integral image pixels at the border is as large as the area covered by the other integral image pixels, the unavailability of pixels beyond the border requires linear interpolation of the border pixels instead of full bilinear interpolation. Resizing at the right border $x=x_{r}$ can be performed by calculating

$$
\begin{array}{r}
I I_{r}\left(x_{r}, y\right) \approx \frac{1}{4 a^{2}} \cdot \operatorname{linear}\left(I I,\left(2 a x_{r}+b, 2 a y+b\right)\right) \\
=\frac{1}{4 a^{2}} \cdot\left((1-d y) \cdot I I\left(\left\lfloor 2 a x_{r}+b\right\rfloor, y^{\prime}\right)+\right. \\
\left.d y \cdot I I\left(\left\lfloor 2 a x_{r}+b\right\rfloor, y^{\prime}\right)\right)
\end{array}
$$

where $y^{\prime}=\lfloor 2 a y+b\rfloor$ and $d y=2 a y+b-y^{\prime}$. Resizing at the bottom border is equivalent for constant $y=y_{b}$ and variable $x$. In case the bottomrightmost pixel cannot be calculated by one of these formulas, it can be approximated without interpolation by

$$
I I_{r}\left(x_{r}, y_{b}\right) \approx \frac{1}{4 a^{2}} \cdot I I\left(\left\lfloor 2 a x_{r}+b\right\rfloor,\left\lfloor 2 a y_{b}+b\right\rfloor\right) .
$$




\section{EVALUATION}

In order to assess the speed, quality and parallelizability of our approach, we created three different implementations in three different languages. Firstly, we created a CUDA program for power-of-two resizing to show the achievable degree of parallelism resulting from the reduced number of memory accesses in this special case (for details see section 4.1). Secondly, we implemented arbitrary resizing in Python including OpenCV's resizing capabilities for comparison to show the quality difference, i.e., the error induced by our approximation. Finally, we modified OpenCV's LBP based (Ahonen et al., 2004) object detection algorithm to use our resizing approach to show the latter's performance and practical use.

All tests were carried out on an Intel Core 2 Duo E6700 desktop system with an NVIDIA GeForce 8500 GT graphics card running Ubuntu 11.1064 bit, unless noted otherwise. We used version 2.4.3 of OpenCV with support for the Intel Thread Building Blocks (TBB) library (version $4.1 \mathrm{Up}-$ date 1).

\subsection{Parallelizability}

For the special case of resizing by a power of two in each dimension, our algorithm for exact bilinear interpolation (see equation (8) requires fewer memory accesses per sample to be calculated (one) than classical bilinar interpolation in the image domain does (four, see equation (4)). Hence, our approach is not slower than classical bilinear interpolation. Additionally, each sample requires a different source integral image sample to be calculated from. Therefore, each sample can be calculated completely independently, allowing for massive parallelization.

Furthermore, if the desired output of the resizing operation is an integral image, classical bilinear interpolation has to be followed by an integral image calculation which is hard to parallelize efficiently, while this is not the case with our approach as its output is another integral image. Thus, a resizing operation with an integral image as final result can be parallelized more easily when using our approach.

To show the latter's parallelizability, we created a straight-forward, unoptimized GPU implementation for resizing an integral image by a factor of two in each dimension in which each image sample is resized by a separate thread calculating

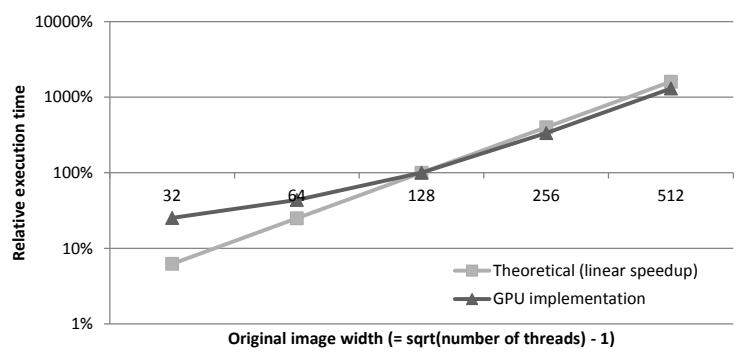

Figure 4: Speedup over number of threads when resizing integral images by a factor of two in each dimension: theoretical linear speedup (light gray rectangles) vs. actual implementation's speedup (dark gray triangles).

equation (9). Given a 32 bits per sample integral image with dimensions $(w+1) \cdot(w+1)$, where $w$ is a power of two, our implementation spawns $\left(\frac{w}{2}+1\right) \cdot\left(\frac{w}{2}+1\right)$ threads on the GPU for calculating the resized integral image.

Note that we did not use the GPU's built-in bilinear resizer in order to keep the implementation as simple as possible. Since the main aim of our implementation is to demonstrate parallelizability, this does not affect the results. Since using the built-in bilinear resizer would only speed up the filtering operation in terms of consumed clock cycles per processed group of pixels, it only differs from the straight-forward implementation by a constant multiplicative factor, which vanishes when considering relative speedup values.

Our implementation's net execution time, i.e., the actual computation time on the GPU, is measured using CUDA events (using CUDA version 4.0 bundled with driver version 304.43 ). In order to avoid the influence of caching effects, before each actual measurement, the GPU kernel is executed three times for cache warming. Subsequently, the actual kernel is executed five times. The average time of these five executions is used to represent the actual net execution time.

Figure 4 depicts the relative net execution time of our resizing approach and a theoretical linear speedup representing the performance of an ideal algorithm with one constant time memory access per sample for comparison. The $x$ axis denotes the values of $w$, while the $y$ axis denotes the speedup relative to the execution time of our GPU implementation's performance for $w=128$ which is the medium measurement point.

As can be seen, the speedup is nearly ideal for larger image dimensions. Although a small overhead remains compared to the theoretically achievable speedup, this is to be expected due to 
the GPU's internal thread scheduling overhead. For smaller image dimensions, the measurements fluctuate significantly due the small number of threads to be executed. Benefitting from the GPU's ability to let multiple threads access the memory at the same time under certain conditions, the achievable speedup for a small number of threads is higher than the simplified theoretical limit and has therefore to be rated with care. However, for a large number of threads this effect becomes relatively small and can therefore be disregarded.

\subsection{Quality}

For arbitrary resizing as described in section 3.1 the quality degradation, i.e., the error introduced by our approach as compared to resizing in the image domain, needs to be assessed. To do so, we use the LIVE (Seshadrinathan et al., 2010) reference picture set and process each picture $I$ in the following way. Firstly, $I$ is resized bilinearly to $I_{r e f}$ with OpenCV to serve as a reference. Secondly, OpenCV is used to compute the integral image of $I$, followed by applying our algorithm for resizing in the integral image domain and subsequently reconstructing the resized image $I_{\text {new }}$ using equation (3). Finally, both, $I_{\text {new }}$ and $I_{r e f}$, are upsampled with nearest neighbour interpolation using OpenCV to fit $I$ 's dimensions and compared to the original image $I$ to determine the respective differences.

Table 1 summarizes the minimum, maximum and average PSNR differences for each resizing factor. Hereby, positive values mean that our approach's PSNR is higher than OpenCV's, while the converse is true for negative values. Although the absolute gap between the minimum and maximum PSNR difference for each factor is not very high in general (around $2 \mathrm{~dB}$ on average), a factordependent trend regarding the average difference can be seen.

While our approach achieves a higher PSNR for large resizing factors (greater than 6.2), the converse is true for small resizing factors (less than 3.1). If little actual interpolation is required (e.g., for factors like 1.0, 2.0 or 3.0), the PSNR differences are smallest on average. Conversely, they amount to up to $4 \mathrm{~dB}$ for quasi-pathological cases like resizing factors of 1.9 .

Although this may seem relatively high, thorough investigation shows that high differences are mainly caused by sub-sample shifts of the image introduced by our algorithm. Interpolating in the integral image domain partly "moves" the area associated with each interpolated column and row to their corresponding neighbours, thereby introducing a sub-pixel shift when reconstructing the image. As this augments the error signal, the PSNR increases. Assuming that most practical applications are not affected by shifts of this magnitude, the quality difference between our bilinear resizer and OpenCV's can be considered acceptably small.

\subsection{Performance}

As state-of-the art object detection algorithms make heavy use of integral images on multiple scales as explained in section 1 , we modified one of them - OpenCV's LBP based object detection algorithm - as an example. Note that this can be done for other multi-scale integral-image-based object detection algorithms in a similar fashion, making the subsequent results applicable to them as well.

While OpenCV's original LBP detector implementation resizes the input image in the image domain and computes its integral image on each scale (see figure 5 left), our modification uses the integral image of the original image and resizes it in the integral image (II) domain (see figure 5 right). The actual detection operations on the resized integral images remain unchanged. However, our modification does not require the integral images to be computed at each scale. Note that this theoretically allows discarding the input picture as soon as the first integral image is calculated. This can save a significant amount of memory, e.g., on embedded systems, when the input image is not needed otherwise. As the default resizing factor used by OpenCV is 1.1 per scale in each dimension, our approximate resizing approach described in section 3.1 is used.

In order to assess the influence of our resizing approach on object detection performance, we trained the LBP detector with OpenCV's face detection training data set and a negative data set from http://note. sonots. com/SciSoftware/haartraining.html. Subsequently, we assessed its detection performance using the four CMU/MIT frontal face test sets from http://vasc.ri.cmu.edu/idb/ images/face/frontal_images. The test data set includes eye, nose and mouth coordinates for each face in each of the 180 pictures. A face is considered detected if and only if all of the aforementioned coordinates are within one of the rect- 
Table 1: Minimum, maximum and average PSNR differences between our approximate resizing approach and OpenCV's bilinear resizer over all pictures of the LIVE data base (Seshadrinathan et al., 2010) for different resizing factors $F$. All PSNR difference values are in dB.

\begin{tabular}{|c|c|c|c|c|c|c|c|c|c|c|c|}
\hline $\mathrm{F}$ & MIN & MAX & $\mathrm{AVG}$ & $\mathrm{F}$ & MIN & MAX & $\mathrm{AVG}$ & $\mathrm{F}$ & MIN & MAX & AVG \\
\hline 1.00 & -0.00 & -0.00 & -0.00 & 4.10 & -1.78 & 0.92 & -0.46 & 7.20 & -0.70 & 1.83 & 0.74 \\
\hline 1.10 & -3.14 & -0.24 & -1.93 & 4.20 & -2.32 & 0.57 & -0.61 & 7.30 & -0.78 & 1.94 & 0.96 \\
\hline 1.20 & -3.14 & 0.26 & -1.57 & 4.30 & -1.15 & 1.10 & -0.01 & 7.40 & -0.44 & 1.58 & 0.68 \\
\hline 1.30 & -3.77 & -1.29 & -2.78 & 4.40 & -1.13 & 0.75 & -0.03 & 7.50 & 0.52 & 2.00 & 1.28 \\
\hline 1.40 & -3.18 & -1.42 & -2.62 & 4.50 & -1.68 & 0.83 & -0.28 & 7.60 & -0.50 & 1.76 & 1.01 \\
\hline 1.50 & -3.10 & 0.16 & -1.10 & 4.60 & -1.49 & 1.06 & -0.07 & 7.70 & -0.77 & 1.37 & 0.62 \\
\hline 1.60 & -2.71 & 0.53 & -0.04 & 4.70 & -1.60 & 1.29 & -0.07 & 7.80 & -0.10 & 1.46 & 0.75 \\
\hline 1.70 & -2.95 & -0.84 & -1.82 & 4.80 & -0.75 & 1.65 & 0.50 & 7.90 & -0.50 & 1.57 & 0.63 \\
\hline 1.80 & -2.47 & -1.29 & -1.94 & 4.90 & -2.14 & 0.95 & -0.25 & 8.00 & -0.47 & 1.86 & 1.48 \\
\hline 1.90 & -4.06 & -0.89 & -2.02 & 5.00 & -0.52 & 2.35 & 0.83 & 8.10 & -0.84 & 1.75 & 0.75 \\
\hline 2.00 & -1.82 & -0.00 & -0.25 & 5.10 & -0.84 & 1.39 & 0.49 & 8.20 & -0.81 & 1.54 & 0.66 \\
\hline 2.10 & -3.45 & -1.73 & -2.58 & 5.20 & -0.86 & 1.11 & 0.34 & 8.30 & -1.04 & 1.25 & 0.37 \\
\hline 2.20 & -3.32 & 0.21 & -1.59 & 5.30 & -1.46 & 1.35 & -0.13 & 8.40 & -1.20 & 1.65 & 0.47 \\
\hline 2.30 & -3.17 & -0.57 & -2.03 & 5.40 & -0.97 & 1.25 & 0.07 & 8.50 & -0.53 & 1.77 & 0.98 \\
\hline 2.40 & -2.17 & 0.58 & -0.53 & 5.50 & -1.36 & 1.42 & 0.40 & 8.60 & -1.08 & 1.39 & 0.56 \\
\hline 2.50 & -2.87 & 0.62 & -1.11 & 5.60 & -0.74 & 1.34 & 0.55 & 8.70 & -0.53 & 1.67 & 0.63 \\
\hline 2.60 & -3.35 & -0.07 & -1.93 & 5.70 & -1.29 & 1.32 & 0.13 & 8.80 & -0.86 & 1.90 & 0.99 \\
\hline 2.70 & -3.06 & 0.53 & -1.46 & 5.80 & -0.10 & 1.59 & 0.80 & 8.90 & -1.13 & 1.49 & 0.65 \\
\hline 2.80 & -2.48 & -0.23 & -1.33 & 5.90 & -0.62 & 1.56 & 0.44 & 9.00 & -0.83 & 2.10 & 0.81 \\
\hline 2.90 & -2.41 & 0.24 & -1.07 & 6.00 & -0.26 & 1.48 & 0.96 & 9.10 & -0.10 & 1.70 & 1.08 \\
\hline 3.00 & -1.57 & 1.82 & -0.08 & 6.10 & -1.57 & 1.40 & -0.15 & 9.20 & -0.33 & 1.77 & 0.90 \\
\hline 3.10 & -1.85 & 0.32 & -0.68 & 6.20 & -0.98 & 1.39 & 0.35 & 9.30 & -0.04 & 1.84 & 1.04 \\
\hline 3.20 & -1.94 & 1.20 & 0.52 & 6.30 & -0.72 & 1.48 & 0.52 & 9.40 & -0.87 & 1.67 & 0.83 \\
\hline 3.30 & -1.98 & 0.94 & -0.32 & 6.40 & -0.17 & 1.83 & 1.17 & 9.50 & -0.93 & 1.48 & 0.42 \\
\hline 3.40 & -2.21 & -0.00 & -0.96 & 6.50 & -1.24 & 1.10 & 0.21 & 9.60 & -0.22 & 2.09 & 1.42 \\
\hline 3.50 & -1.51 & 0.65 & -0.19 & 6.60 & -1.10 & 1.82 & 0.52 & 9.70 & -1.07 & 1.91 & 0.84 \\
\hline 3.60 & -1.41 & 0.81 & 0.13 & 6.70 & -0.31 & 1.32 & 0.50 & 9.80 & -0.54 & 1.90 & 1.02 \\
\hline 3.70 & -1.92 & 0.43 & -0.55 & 6.80 & -1.26 & 1.57 & 0.33 & 9.90 & -0.69 & 1.81 & 0.65 \\
\hline 3.80 & -1.77 & 0.32 & -0.39 & 6.90 & -0.22 & 1.83 & 0.84 & 10.00 & -0.64 & 1.90 & 1.13 \\
\hline 3.90 & -1.77 & 0.91 & -0.27 & 7.00 & -1.21 & 1.60 & 0.56 & & & & \\
\hline 4.00 & -1.07 & 1.08 & 0.72 & 7.10 & -0.73 & 1.98 & 1.10 & & & & \\
\hline
\end{tabular}

angles returned by the LBP based detector. The detection rate is determined as the ratio of the number of detected faces to the total number of faces.

In total, the detection rate does not change, i.e., both, OpenCV's and our modification's, detection rates are exactly the same, namely $46.19 \%$. It should be noted that not all detected faces coincide completely, i.e., the detected rectangles differ slightly due to the sub-pixel shift introduced by our approach on smaller scales as explained in section 4.2 . In addition, $15 \%$ of all pictures exhibit differences in the number of detected faces, which is mainly due to fact that the detector's training was performed using regularly resized training data. We conjecture that, when using our resizing approach during training as well, the aforemen- tioned differences will possibly vanish.

In order to assess the performance gain of our modification in terms of execution time in a fair way, we do not perform execution time measurements for our unoptimized modification and the highly optimized original OpenCV code. Instead, we deduce the performance gain as follows: As our resizing approach in the integral image domain is identical to bilinear interpolation in the image domain in terms of operations (see section 3.1), our modification does not impact the resizing speed. Conversely, as our approach does not require integral image calculations at any scale but the first (see above), the remaining integral image calculations do not need to be performed. Therefore, the execution time of these integral image calculations relative to the detector's to- 

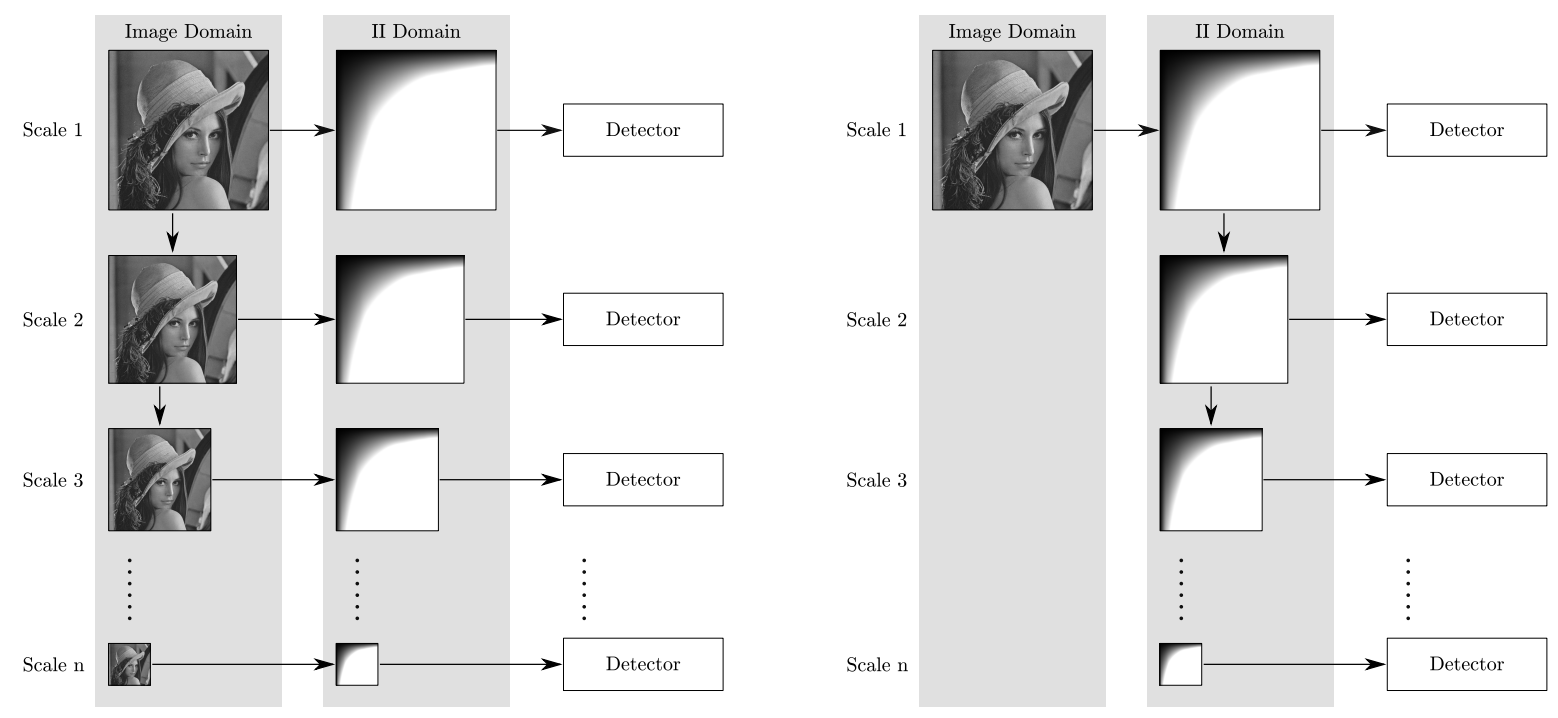

Figure 5: Illustration and comparison of OpenCV's multi-scale LBP detector (left) and our modification of it (right). By using the proposed integral image resizing approach, our modification does not require the recalculation of the integral images on each scale.

tal execution time is equivalent to the potential speedup of our approach compared to the current OpenCV implementation.

For the accurate measurement of single functions' execution times, rdtsc (Intel, 2012) commands are placed before and after the corresponding function calls inside the OpenCV code. We use the aforementioned CMU/MIT test set and execute the detector a total of 110 times for each image - ten times for cache warming and 100 times for the actual time measurement as described in section 4.1. To address the question of scalability, two additional test systems with comparable software configurations are used for this evaluation: a mobile system (henceforth referred to as system B) with an Intel Core i5 540M CPU with two physical cores capable of hyper-threading, i.e., a total of four virtual CPU cores, and a server system (henceforth referred to as system C) with 4 AMD Opteron 6274 CPUs with 16 cores each, i.e., a total of 64 physical CPU cores.

The results vary strongly depending on three parameters: the image size, the image content and the number of available CPU cores. The former two parameters influence the number of actual resizing operations being performed, yielding different speedups for different picture sizes and content types. As the default resizing factor per scale is 1.1 in each dimension, larger images with big objects to be detected exhibit a larger speedup than smaller images do. Similarly, when large image areas without detectable objects are present, the speedup is greater as more execution time is spent in the integral image calculation routines than in the actual detector due to the LBP cascades on each scale terminating quickly.

The influence of the third and most influential parameter, i.e., the number of available $\mathrm{CPU}$ cores, is summarized in table 2, It shows that the default test system with two CPU cores (referred to as system A) spends on average $4.64 \%$ of the detector's execution time on the described integral image calculations, which is equivalent to an average speedup of $4.64 \%$ of our proposed modification compared to the existing OpenCV implementation (see above). Using the four virtual cores of test system B, the speedup increases to an average of $6.38 \%$. This is due to the fact that the integral image calculations cannot be parallelized efficiently, while the converse is true for most of the detector's other code parts. Thus, our proposed modification using our integral image based resizing approach provides better scalability. This becomes even clearer when considering test system $\mathrm{C}$ with 64 total CPU cores with an average and maximum speedup of $12.6 \%$ and $37.25 \%$, respectively.

Note that our modification also allows speeding up the overall detection process when TBB support in OpenCV is disabled, i.e., when only one CPU core is actually used. This can be seen in the corresponding row in table 2 which reveals an average speedup of $2.9 \%$ in this case. However, it is not recommended to only use one CPU core 
Table 2: Dependency of integral image calculation time at lower scales of the LBP detector on the number of available CPU cores $n$. All values are relative to the detector's total execution time for the respective hardware and software configuration.

\begin{tabular}{|l|l|l|l|l|l|}
\hline$n$ & SYS & AVG & SDEV & MIN & MAX \\
\hline 1 & A $^{*}$ & $2.9 \%$ & $0.71 \%$ & $1.57 \%$ & $6.78 \%$ \\
\hline 2 & A & $4.66 \%$ & $0.66 \%$ & $2.92 \%$ & $6.89 \%$ \\
\hline 4 & B $^{* *}$ & $6.38 \%$ & $0.78 \%$ & $4.38 \%$ & $9.87 \%$ \\
\hline 64 & C & $12.6 \%$ & $4.86 \%$ & $4.21 \%$ & $37.25 \%$ \\
\hline \multicolumn{6}{|c|}{ TBB support disabled } \\
2 cores with hyper-threading
\end{tabular}

when more are available as a higher number of CPU cores increases the speedup significantly as shown above.

\section{CONCLUSION}

We proposed a new approach for image resizing which works entirely in the integral image domain. For the special case of power-of-two resizing, we presented a highly parallelizable version of our approach which requires only a quarter of the operations compared to regular bilinear interpolation in the image domain, but provides the same exact results. Furthermore, we evaluated the practicality of our general approach by modifying one of multiple state-of-the-art multi-scale integral-image-based object detection algorithms in OpenCV without degrading its detection performance. In total, a speed-up of an average of $6.38 \%$ and $12.6 \%$ could be achieved on a dual-core mobile computer and a multi-processor server, respectively. Moreover, we showed that similar results can be achieved for all multi-scale integralimage-based object detection algorithms.

\section{ACKNOWLEDGEMENTS}

This work is supported by FFG Bridge project 832082 .

\section{REFERENCES}

Ahonen, T., Hadid, A., and Pietikäinen, M. (2004). Face Recognition with Local Binary Patterns. In Pajdla, T. and Matas, J., editors, Computer Vision - ECCV 2004, volume 3021 of Lecture Notes in Computer Science, pages 469-481. Springer Berlin Heidelberg.

Bay, H., Ess, A., Tuytelaars, T., and Van Gool, L. (2008). Speeded-up robust features (surf). Comput. Vis. Image Underst., 110:346-359.

Bilgic, B., Horn, B. K., and Masaki, I. (2010). Efficient integral image computation on the GPU. In 2010 IEEE Intelligent Vehicles Symposium (IV), pages 528-533, San Diego, CA, USA. IEEE.

Crow, F. C. (1984). Summed-area tables for texture mapping. In Proceedings of the 11th annual conference on Computer graphics and interactive techniques, SIGGRAPH '84, pages 207-212, New York, NY, USA. ACM.

Heckbert, P. S. (1986). Filtering by repeated integration. In Proceedings of the 13th annual conference on Computer graphics and interactive techniques, SIGGRAPH '86, pages 315-321, New York, NY, USA. ACM.

Hensley, J., Scheuermann, T., Coombe, G., Singh, M., and Lastra, A. (2005). Fast Summed-Area Table Generation and its Applications. Computer Graphics Forum, 24(3):547-555.

Hussein, M., Porikli, F., and Davis, L. (2008). Kernel integral images: A framework for fast non-uniform filtering. In IEEE Conference on Computer Vision and Pattern Recognition 2008 (CVPR 2008), pages 1-8, Anchorage, AK, USA. IEEE.

Intel (2012). Intel 64 and IA-32 Architectures Software Developer's Manual, Volume 2B: Instruction Set Reference, N-Z. http://www.intel. com/Assets/PDF/manual/253667.pdf

Seshadrinathan, K., Soundararajan, R., Bovik, A., and Cormack, L. (2010). Study of Subjective and Objective Quality Assessment of Video. IEEE Transactions on Image Processing, 19(6):14271441.

Viola, P. and Jones, M. (2001). Robust Real-time Object detection. In International Journal of Computer Vision, volume 57, pages 137-154.

Willow Garage (2012). OpenCV. http://opencv. willowgarage.com/

$\mathrm{Yu}, \mathrm{C}$., Dian-ren, C., Xu, Y., and Yang, L. (2010). Fast Two-Dimensional Otsu's Thresholding Method Based on Integral Image. In 2010 International Conference on Multimedia Technology (ICMT), pages 1-4, Ningbo, China. IEEE. 